\title{
Does Measurement of Esophagogastric Junction Distensibility by EndoFLIP Predict Therapy- responsiveness to Endoluminal Fundoplication in Patients With Gastroesophageal Reflux Disease?
}

\author{
Fabiënne G M Smeets, ${ }^{1,2}$ Daniel Keszthelyi, ${ }^{1,2}$ Nicole D Bouvy, ${ }^{3}$ Ad A M Masclee ${ }^{1,2}$ and José M Conchillo ${ }^{1,2 *}$ \\ Departments of ${ }^{1}$ Gastroenterology and Hepatology, and ${ }^{3}$ General Surgery, Maastricht University Medical Center, Maastricht, the Netherlands; \\ and ${ }^{2}$ NUTRIM, School of Nutrition and Translational Research in Metabolism, Maastricht University, Maastricht, the Netherlands
}

\section{Background/Aims}

In patients with gastroesophageal reflux disease (GERD), an increased esophagogastric junction (EGJ) distensibility has been described. Assessment of EGJ distensibility with the endoscopic functional luminal imaging probe (EndoFLIP) technique might identify patients responsive to transoral incisionless fundoplication (TIF), whereas postoperative measurement of EGJ distensibility might provide insight into the antireflux mechanism of TIF. Therefore, we investigated the value of the EndoFLIP technique in GERD patients treated by TIF.

\section{Methods}

Forty-two GERD patients underwent EGJ distensibility measurement before TIF using the EndoFLIP technique. In a subgroup of 25 patients, EndoFLIP measurement was repeated both postoperative and at 6 months follow-up. Treatment outcome was assessed according to esophageal acid exposure time (AET; objective outcome) and symptom scores (clinical outcome) 6 months after TIF.

\section{Results}

Multiple logistic regression analysis showed that preoperative $E G J$ distensibility $(O R, 0.16 ; 95 \% C l, 0.03-0.78 ; P=0.023)$ and preoperative $A E T(O R, 0.62 ; 95 \% \mathrm{Cl}, 0.42-0.90 ; P=0.013)$ were independent predictors for objective treatment outcome but not for clinical outcome after TIF. The best cut-off value for objective outcome was $2.3 \mathrm{~mm}^{2} / \mathrm{mmHg}$ for preoperative EGJ distensibility and $11 \%$ for preoperative AET. EGJ distensibility decreased direct postoperative from 2.0 (1.2-3.3) to 1.4 (1.0-2.2) $\mathrm{mm}^{2} / \mathrm{mmHg}(P=0.014)$, but increased to $2.2(1.5-3.0)$ at 6 months follow-up ( $P=0.925$, compared to preoperative).

Received: September 24, 2014 Revised: November 11, 2014 Accepted: November 12, 2014

(c) This is an Open Access article distributed under the terms of the Creative Commons Attribution Non-Commercial License (http://creativecommons. org/licenses/by-nc/3.0) which permits unrestricted non-commercial use, distribution, and reproduction in any medium, provided the original work is properly cited.

*Correspondence: José M Conchillo, MD

Department of Gastroenterology and Hepatology, Maastricht University Medical Center, Postbox 5800, P. Debyelaan 25, 6229 HX Maastricht, the Netherlands

Tel: +3143-3875021, Fax: +3143-3875006, E-mail: j.conchillo@mumc.nl

Financial support: None.

Conflicts of interest: None.

Author contributions: Fabiënne G M Smeets, analysis and interpretation of data, and drafting of the manuscript; Daniel Keszthelyi, analysis and interpretation of data, and critical revision of the manuscript for important intellectual content; Nicole D Bouvy, conducting the study, and critical revision of the manuscript for important intellectual content; Ad A M Masclee, critical revision of the manuscript for important intellectual content; José M Conchillo, conducting the study, analysis and interpretation of data, drafting and critical revision of the manuscript for important intellectual content, and final approval. 


\section{Conclusions}

Preoperative EGJ distensibility and preoperative AET were independent predictors for objective treatment outcome but not for clinical outcome after TIF. According to our data, the EndoFLIP technique has no added value either in the preoperative diagnostic work-up or in the post-procedure evaluation of endoluminal antireflux therapy.

(J Neurogastroenterol Motil 2015;21:255-264)

\section{Key Words}

Esophagogastric Junction; Fundoplication; Gastroesophageal reflux disease; Treatment outcome

\section{Introduction}

Pharmacological therapy with proton pump inhibitors (PPIs) is effective in controlling reflux symptoms and therefore considered the mainstay for gastroesophageal reflux disease (GERD) treatment. However, in case of unresponsiveness to PPIs or incomplete symptom relief, antireflux surgery should be considered. ${ }^{1}$ Antireflux surgery controls GERD by restoring the competence of the gastroesophageal junction. It should be taken into account that antireflux surgery is associated with short and long-term adverse effects including dysphagia and gas bloat. ${ }^{2}$ In the last decade, several endoscopic treatment options have been developed as less invasive alternatives to antireflux surgery. ${ }^{3}$ Most of the devices like Gatekeeper ${ }^{\mathrm{tm}}$ and Enteryx showed acceptable to good short-term outcomes but are no longer available due to poor long-term outcomes and the occurrence of complications. ${ }^{4}$ Transoral incisionless fundoplication (TIF) using the EsophyX device is a novel technique developed for treatment of GERD patients and previous studies demonstrated that TIF is a safe treatment option and effective in improving quality of life. ${ }^{5}$ However, in uncontrolled trials normalization of acid exposure time is only present in $37-50 \%$ of patients with a lack of correlation between objective and subjective treatment outcomes assessed with $\mathrm{pH}$-metry and validated questionnaires. ${ }^{6-8}$ Previous studies have attempted to identify patient characteristics and objective parameters like lower esophageal sphincter (LES) resting pressure and the number of fasteners that may help to predict treatment outcome of the TIF procedure. ${ }^{9,10}$ However, up to now, no objective parameter is available to select patients for this endoluminal procedure.

Increased esophagogastric junction (EGJ) distensibility is considered a pathophysiologic factor in GERD as EGJ compliance determines the opening diameter of the EGJ and consequently the volume of reflux content into the esophagus. ${ }^{11,12}$
Surgical treatment options attempt to restore anatomic components of the antireflux barrier and previous studies using the barostat method have described a reduction in EGJ distensibility after laparoscopic Nissen fundoplication. ${ }^{13,14}$ Recently, a new endoscopic functional luminal imaging probe (EndoFLIP) has been developed to assess EGJ distensibility using impedance planimetry during volumetric distensions. Advantages of this technique compared to barostat are its use without need for additional fluoroscopy, real time dynamic imaging of the EGJ and fast assessment of EGJ distensibility patterns. ${ }^{15}$ Stratification of GERD patients according to EGJ distensibility might help to identify patients responsive to TIF and we hypothesize that patients with low preoperative EGJ distensibility will have better EGJ competence and consequently a better response to TIF compared to patients with high EGJ distensibility. Moreover, the use of the EndoFLIP in the postoperative period might provide more insight into the antireflux mechanism of this endoluminal fundoplication procedure. ${ }^{15}$ Therefore, we investigated the value of the EndoFLIP technique in GERD patients treated by an endoluminal fundoplication procedure.

\section{Materials and Methods}

\section{Patients}

Between 2009 and 2012, transoral incisionless fundoplication was performed in 60 chronic GERD patients in a randomized controlled trial comparing TIF with acid suppressive medication at the Maastricht University Medical Center. Outcomes of this randomized controlled trial from our group are awaited with long-term data after endoluminal fundoplication (including safety, complications, and need for additional treatment) and will be reported separately. In the present study, a post-hoc analysis was performed in 42 patients to investigate the value of the EndoFLIP technique in endoluminal antireflux surgery. 
Patients were referred for antireflux surgery either because they were refractory, unsatisfied or unwilling to take lifelong acid suppressive medication. Patients underwent upper gastrointestinal endoscopy, 24-hour $\mathrm{pH}$-impedance monitoring and esophageal manometry as standard preoperative work-up for antireflux surgery.

Patients with chronic GERD symptoms ( $>6$ months), esophagitis at upper gastrointestinal endoscopy and/or abnormal acid exposure time during 24-hour $\mathrm{pH}$-impedance monitoring ( $\mathrm{pH}<4$ during $>4 \%$ of time) were included. All patients were aged 18 to 75 years with a body mass index (BMI) below 35 $\mathrm{kg} / \mathrm{m}^{2}$ and normal LES resting pressure $(<30 \mathrm{mmHg})$. Exclusion criteria were age $<18$ years, current pregnancy, severe reflux esophagitis grade D according to the Los Angeles classification, other esophageal diseases (including esophageal motility disorders, biopsy proven Barrett's esophagus, esophageal stricture or esophageal ulcer), previous antireflux surgery or severe comorbidities (including cardiopulmonary disease, portal hypertension, coagulation disorders, immunosuppression or morbid obesity). Patients with hiatal hernia $>2 \mathrm{~cm}$ were excluded, since previous studies demonstrated that complete reduction of hernias was more often achieved in patients with small hiatal hernia (1-2 $\mathrm{cm})$ compared to patients with large hiatal hernia $(>3 \mathrm{~cm}) .{ }^{10}$

The protocol was approved by the medical ethical committee of the Maastricht University Medical Center (METC 07-2-051, NL17303.068.07) and was registered NCT00857597 (clinicaltrials.gov). Written informed consent was obtained from all patients.

\section{Study Protocol}

Symptom scores, upper gastrointestinal endoscopy and 24-hour $\mathrm{pH}$-impedance monitoring were performed preoperatively to assess whether patients were eligible for the TIF procedure and these measurements were repeated 6 months after the endoluminal fundoplication. Esophageal manometry was performed preoperatively to exclude esophageal motility disorders. All patients underwent one EndoFLIP measurement before the TIF procedure and EndoFLIP measurements were repeated in the direct postoperative state and at 6 months follow-up in the last 25 consecutive patients treated with TIF.

\section{Assessment of Symptoms and Medication Use}

Symptoms were assessed using the GERD health-related quality of life (GERD-HRQL), a validated questionnaire consisting of 10 questions measuring heartburn, dysphagia, bloating, and satisfaction with the impact of medication on daily life, with scores from 0 (no symptoms) to 5 (worst symptoms). ${ }^{16}$ Total GERD-HRQL scores were calculated by summing the individual items with a maximal score of 50 points. Symptoms were evaluated at baseline while off acid suppressive medication and at 6 months follow-up. Clinical treatment outcome was assessed 6 months after TIF and patients achieving at least $50 \%$ improvement in GERD-HRQL scores compared to baseline off PPIs were considered responsive to the procedure. ${ }^{17}$ Use of acid suppressive medication was assessed preoperative and 6 months after TIF. The use of acid suppressive medication was recorded and categorized in none, occasional or common usage. "Common" usage of PPIs corresponded to full dose or half dose taken daily for more than $50 \%$ of the follow-up period, "occasional” usage of PPIs was defined as less than a half dose or any daily dose of PPIs taken for less than $50 \%$ of total number of follow-up days, and "none" when patients did not use PPIs in the preceding follow-up period.

\section{Endoscopy}

Upper endoscopy was performed by a single gastroenterologist prior to the TIF procedure (JC). Patients underwent endoscopy in the left lateral decubitus position after induction of general anesthesia. Presence and size of hiatal hernia, and reflux esophagitis according to the Los Angeles classification were determined. In retroversion, the gastroesophageal junction was assessed according to the Hill classification (grade I-IV). Patients with Hill grade I or II were considered as patients with normal gastroesophageal flap valve grade and patients with Hill grade III or IV as patients with abnormal flap valve. ${ }^{18}$

\section{Ambulatory 24-hour pH-Impedance Monitoring}

Ambulatory 24-hour $\mathrm{pH}$-impedance monitoring was performed at baseline and 6 months after TIF using a combined pH-impedance catheter (Unisensor AG, Attikon, Switzerland). The catheter was introduced transnasally and contained six impedance segments and one $\mathrm{pH}$-electrode. Impedance recording segments were located 2-4, 4-6, 6-8, 8-10, 14-16, and 16-18 cm above the upper border of the LES and the $\mathrm{pH}$ electrode was positioned $5 \mathrm{~cm}$ above the upper border of the LES, as determined during esophageal manometry. The $\mathrm{pH}$-impedance catheter was left in situ for 24 hours and data from the catheter were transmitted to and recorded on a portable recorder (Ohmega, Medical Measurement Systems, Enschede, The Netherlands). Patients received a personal diary to note GERD 
symptoms, meal periods and body position (upright or supine), meal periods were excluded from the analysis.

Analysis of 24-hour pH-impedance measurements included acid exposure time (AET, \%) and number of liquid, proximal and acid reflux episodes. Reflux episodes with $\mathrm{pH}<4$ were classified as acidic reflux episodes and reflux episodes with $\mathrm{pH} \geq 4$ were classified as non-acidic reflux episodes. Reflux episodes reaching the 2 proximal recording segments were considered as proximal reflux episodes. Objective treatment outcome was assessed according to AET at 6 months follow-up. Patients with normalized AET ( $\mathrm{pH}<4$ for $\leq 4 \%$ of time) were considered as patients with a good objective treatment outcome whereas patients with persistent abnormal AET $(\mathrm{pH}<4$ during $>4 \%$ of time) were considered as patients with a poor objective treatment outcome.

\section{Assessment of Esophagogastric Junction Distensibility}

Esophagogastric junction distensibility was measured using a commercially available functional luminal imaging probe (EndoFLIP; Crospon Medical Devices, Galway, Ireland). ${ }^{15,19}$ The EndoFLIP probe consists of a catheter with a noncompliant bag on its distal end, which encloses 17 electrodes at $5 \mathrm{~mm}$ intervals. Cross sectional areas (CSAs) are determined for 16 balloon cross sections during volumetric distensions using impedance planimetry. One pressure sensor is located on the probe to determine intra-bag pressure allowing assessment of EGJ distensibility of the distended area. Baseline pressure was zeroed prior to insertion of the catheter. At the end of endoscopy the deflated catheter was inserted transorally and positioned at the EGJ. The endoscope was withdrawn and the balloon was inflated to 20 $\mathrm{mL}$ and $30 \mathrm{~mL}$ distension volumes with a specially formulated conductive solution. To ensure proper bag placement, EndoFLIP measurements were monitored in real time by the display on the recording unit. An hourglass shape of the balloon was observed in all measurements with the narrowest CSA at the level of the esophagogastric junction. Measurements interrupted by balloon migration or esophageal peristalsis were repeated. After completing EGJ measurements, the EndoFLIP bag was deflated and removed. Immediately after the TIF procedure a second EndoFLIP measurement was performed in 25 patients. EGJ distensibility was assessed using the mean value over a 30 seconds dynamic measurement of the narrowest CSA and the corresponding intra-bag pressure (narrowest CSA in $\mathrm{mm}^{2}$ /intra-bag pressure in $\mathrm{mmHg}$ ).

\section{Transoral Incisionless Fundoplication Procedure}

TIF was performed using the EsophyX-2 device (EndoGastric Solutions, Inc, Redmond, WA, USA) according to the protocol as previously described. ${ }^{20}$ Patients were placed in the left lateral decubitus position after induction of general anesthesia. All procedures were performed by an experienced team of a gastroenterologist and a surgeon. The EsophyX device was inserted transorally over a flexible endoscope into the stomach. A retractor was anchored at the gastroesophageal junction and a hiatal hernia, if present, was reduced by returning the squamocolumnar junction to its natural position below the diaphragm using a built-in vacuum invaginator. A partial fundoplication was constructed through sequential retractions of tissue and tailored placement of multiple H-shaped polypropylene fasteners circumferentially around the gastroesophageal junction to create a full thickness 240-330 degree circumference fundoplication (Fig. 1).

\section{Statistical Methods}

Data for continuous variables are expressed as median (interquartile range $[\mathrm{IQR}])$. Statistical comparisons between preoperative and postoperative parameters were performed using Wilcoxon signed rank test; in case of proportions, McNemar's
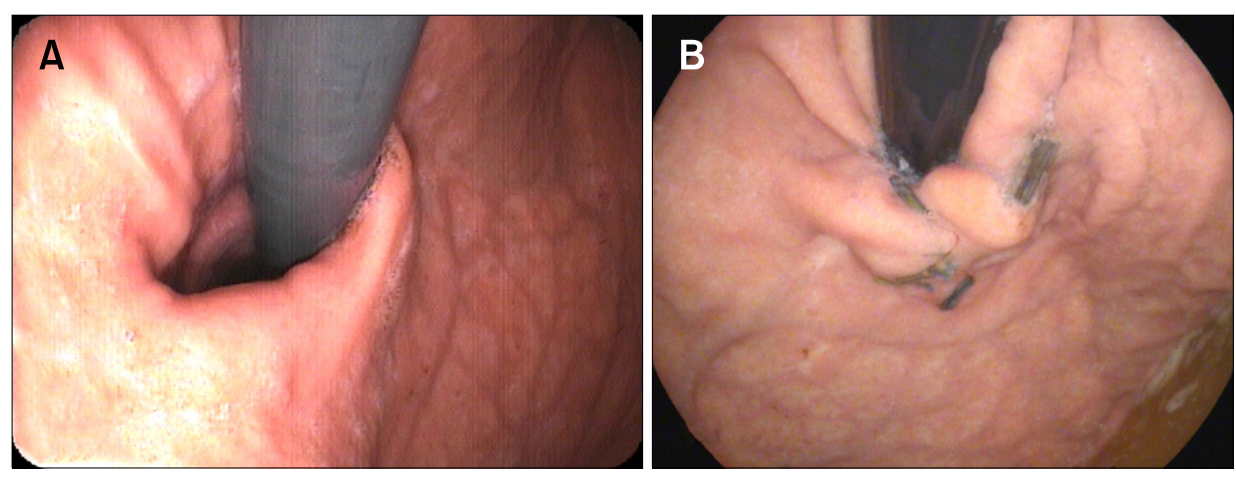

Figure 1. Gastroesophageal flap valve before (A) and after (B) transoral incisionless fundoplication. 
test was used. The Mann-Whitney $\mathrm{U}$ test was used to assess differences between patients with a good or poor treatment outcome according to esophageal AET (objective outcome) or GERD-HRQL score (clinical outcome) at 6 months follow-up. Categorical variables were assessed using Chi-square test and correlations between EGJ distensibility and $\mathrm{pH}$-impedance parameters were calculated using Spearman's correlation coefficient. Multiple logistic regression analysis using age, gender, preoperative EGJ distensibility, preoperative acid exposure time, presence or absence of hiatal hernia and gastroesophageal flap valve grade (normal or abnormal flap valve grade) was performed to identify predictors of treatment success for the TIF procedure. Odds ratios (OR) are presented with 95\% confidence intervals (CI). Values of $P<0.05$ were considered significant. All statistical analyses were performed using commercially available computer software (PASW Statistics for Windows, Release Version 20.0.0; SPSS Inc, Chicago, IL, USA).

\section{Results}

\section{Patient Characteristics and Symptom Scores}

Of the total of 42 patients, 26 were male $(62 \%)$, mean age was 45 years (range, 20-68 years) with a median BMI of 25.6 $\mathrm{kg} / \mathrm{m}^{2}\left(23.5-27.9 \mathrm{~kg} / \mathrm{m}^{2}\right)$. Assessment of EGJ distensibility using the EndoFLIP system was feasible in all but one patient due to incorrect placement of the EndoFLIP balloon and no adverse events occurred. Six months after TIF, symptom scores and 24-hour $\mathrm{pH}$-impedance monitoring results were available for 37 patients, whereas upper endoscopy was performed in 38 patients. Three patients were lost to follow-up, one patients had a defective $\mathrm{pH}$-measurement recording and one patient refused to undergo pH-impedance monitoring due to a psychiatric disorder.

Six months after TIF, GERD symptoms had improved according to the reduction in GERD-HRQL scores (from 26
[21-35]) to 10 [4-20], $P<0.01)$. Common use of acid suppressive medication was discontinued by $67 \%$ of patients, whereas $19 \%$ used PPIs occasionally and common use was present in $14 \%$.

\section{Endoscopy}

Prior to the TIF procedure, hiatal hernia was present in 28 (67\%) patients ( $1 \mathrm{~cm}: 11$ patients; $2 \mathrm{~cm}: 17$ patients), most patients had gastroesophageal flap valve grade II $(60 \%)$ or III (31\%) and in 12 (29\%) patients esophagitis was described (grade A: 5 patients; grade B: 6 patients; grade C: 1 patient).

Six months after TIF, hiatal hernia was present in 15 (39\%) patients $(1 \mathrm{~cm}: 7$ patients; $2 \mathrm{~cm}: 8$ patients; $P=0.052$, compared to preoperative), $82 \%$ of patients had a normal Hill grade $(P=$ 0.022 , compared to preoperative) and $8(21 \%)$ patients had esophagitis (grade A: 3 patients; grade B: 4 patients; grade C: 1 patient). Preoperative esophagitis was cured in 8 patients, whereas 4 patients had persistent esophagitis and 4 patients had esophagitis de novo $(P=0.388)$.

\section{Objective Treatment Outcome}

Esophageal acid exposure time (AET) and number of reflux episodes were reduced at 6 months follow-up (Table 1). Normalization of esophageal AET was achieved in 21 patients whereas 16 patients had persistent abnormal AET. No difference was found in symptom scores between patients with normalized and persistent abnormal AET both preoperatively and at 6 months follow-up, and both patient groups had a significant reduction in symptom scores after TIF ( $P<0.01$, compared to preoperative). Six months after TIF, no difference was found in medication use between both patient groups ( $P=0.677$ ), whereas normal Hill grade was more often present in patients with normalized AET compared to patients with persistent pathological AET (100\% vs $56 \%, P<0.01)$.

Patients with normalization of AET at 6 months follow-up

Table 1. Twenty-four Hour $\mathrm{pH}$-impedance Parameters Before and 6 Months After Transoral Incisionless Fundoplication $(\mathrm{N}=37)$

\begin{tabular}{lccc}
\hline & Preoperative & Six months follow-up & $P$-value \\
\hline Acid exposure time total (\%) & $9.7(5.6-13.7)$ & $3.8(2.0-9.0)$ & $<0.01$ \\
Acid exposure time upright (\%) & $13.1(6.6-18.1)$ & $5.8(3.4-11.0)$ & $<0.01$ \\
Acid exposure time supine (\%) & $4.4(0.7-10.3)$ & $0.1(0.0-3.2)$ & $<0.05$ \\
Liquid reflux episodes (n) & $90(70-114)$ & $62(51-90)$ & $<0.01$ \\
Acid reflux episodes (n) & $59(43-76)$ & $36(21-50)$ & $<0.01$ \\
Proximal reflux episodes (n) & $45(31-53)$ & $23(14-37)$ & $<0.01$ \\
\hline
\end{tabular}

Values are presented as median (IQR). 
turned out to have a lower preoperative esophageal AET and lower preoperative EGJ distensibility compared to patients with persistent abnormal AET (Table 2). Six months after TIF, patients with normalized AET had lower numbers of liquid, acid and proximal reflux episodes compared to patients with persistent pathological AET (liquid: 57 [36-88] vs 80 [60-129], $P=$ 0.012 ; acid: 31 [16-37] vs 58 [35-68], $P<0.01$; proximal: 19 [8-27] vs 41 [21-56], $P<0.01)$.

Multiple logistic regression analysis, adjusted for age and gender, showed that preoperative EGJ distensibility (in $\mathrm{mm}^{2} /$ $\mathrm{mmHg}$; OR 0.16 , 95\% CI 0.03-0.78; $P=0.023$ ) and preoperative AET (in \%; OR 0.62, 95\% CI 0.42-0.90; $P=0.013$ )

Table 2. Preoperative EndoFLIP Parameters and Preoperative Acid Exposure Time in Patients With Normalized $(\mathrm{pH}<4$ for $\leq 4 \%$ of Time) and Patients With Persistent Abnormal Acid Exposure Time at 6 Months Follow-up

\begin{tabular}{lccc}
\hline & $\begin{array}{c}\text { Normalized AET } \\
(\mathrm{n}=21)\end{array}$ & $\begin{array}{c}\text { Abnormal AET } \\
(\mathrm{n}=16)\end{array}$ & $P$-value \\
\hline $\begin{array}{l}\text { EGJ distensibility } \\
\left(\mathrm{mm}^{2} / \mathrm{mmHg}\right)\end{array}$ & & & \\
$20 \mathrm{~mL}$ & $1.3(1.0-1.7)$ & $2.1(1.2-2.4)$ & 0.060 \\
$30 \mathrm{~mL}$ & $1.5(1.1-2.1)$ & $2.5(1.7-3.4)$ & $<0.05$ \\
CSA $\left(\mathrm{mm}^{2}\right)$ & & & \\
$20 \mathrm{~mL}$ & $21(20-24)$ & $25(21-33)$ & $<0.05$ \\
$30 \mathrm{~mL}$ & $29(22-38)$ & $38(26-54)$ & $<0.05$ \\
Pressure $(\mathrm{mmHg})$ & & & $\mathrm{NS}$ \\
$20 \mathrm{~mL}$ & $16(12-21)$ & $13(11-18)$ & $\mathrm{NS}$ \\
$30 \mathrm{~mL}$ & $20(15-26)$ & $15(14-21)$ & \\
Acid exposure $(\%)$ & & & \\
Total & $7.6(5.3-10.6)$ & $11.8(9.3-16.8)$ & $<0.05$ \\
Upright & $7.9(5.8-15.4)$ & $15.4(13.1-25.3)<0.05$ \\
Supine & $4.3(0.3-10.3)$ & $5.0(1.2-12.9)$ & $\mathrm{NS}$ \\
\hline
\end{tabular}

AET, acid exposure time; EGJ, esophagogastric junction; CSA, cross sectional area; NS, not significant.

Values are presented as median (IQR). were independent predictors for objective treatment success (ie, normalization of acid exposure time) after TIF, whereas presence of preoperative hiatal hernia and gastroesophageal flap valve grade could not predict objective treatment outcome after TIF (Table 3). Since preoperative EGJ distensibility and preoperative AET are predictors of objective treatment outcome, we determined cut-off values to improve patient selection for the TIF procedure. To assess the best cut-off values, we evaluated the percentage of patients with normalized or persistent abnormal AET for different values. The best cut-off value for preoperative EGJ distensibility $(30 \mathrm{~mL})$ was $2.3 \mathrm{~mm}^{2} / \mathrm{mmHg}$ as patients with a lower distensibility had normalized AET in $72 \%$ of cases, while patients with a higher preoperative distensibility maintained an abnormal AET in $82 \%$ of cases. The optimal cut-off value for preoperative AET was $11 \%(<11 \%: 77 \%$ had normalized AET; $>11 \%: 79 \%$ had persistent abnormal AET). Combining these

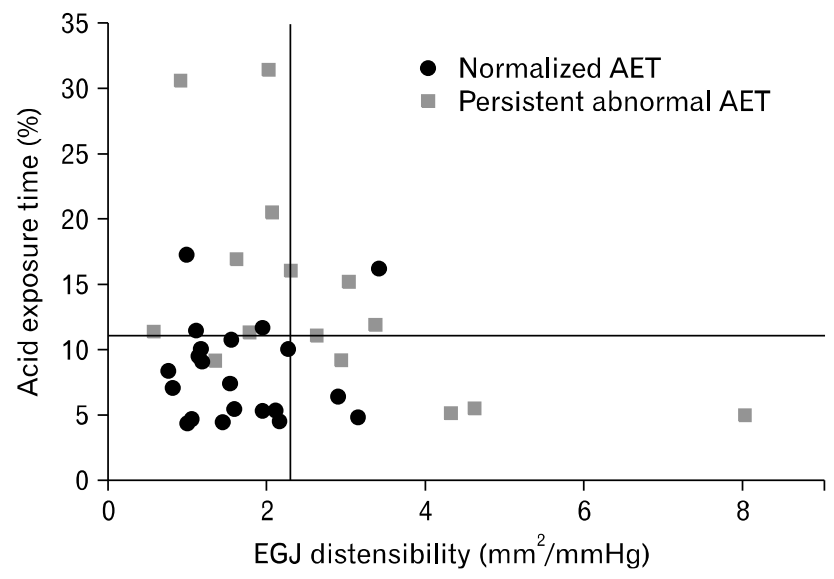

Figure 2. Relationship between preoperative esophagogastric junction (EGJ) distensibility and preoperative acid exposure time (AET) in patients with normalized AET $(\mathrm{n}=21)$ and persistent abnormal acid exposure time $(n=16)$ at 6 months follow-up. Cut-off values: EGJ distensibility, $2.3 \mathrm{~mm}^{2} / \mathrm{mmHg}$; acid exposure time, $11 \%$.

Table 3. Multiple Logistic Regression Analysis to Examine Preoperative Predictors for Normalization of Acid Exposure Time at 6 Months Follow-up

\begin{tabular}{lllc}
\hline \multicolumn{1}{c}{ Parameters } & OR & 95\% CI & $P$-value \\
\hline Female gender (vs male gender) & 2.69 & $0.18-39.75$ & 0.471 \\
Age (yr, continuous) & 0.99 & $0.89-1.10$ & 0.816 \\
EGJ distensibility (mm ${ }^{2} / \mathrm{mmHg}^{\prime}$, continuous) & 0.16 & $0.03-0.78$ & 0.023 \\
Acid exposure time $(\%$, continuous) & 0.62 & $0.42-0.90$ & 0.013 \\
Normal gastroesophageal flap valve grade (vs abnormal flap valve grade) & 0.30 & $0.02-3.70$ & 0.345 \\
Absence of hiatal hernia (vs presence of small hiatal hernia) & 1.52 & $0.14-16.34$ & 0.731 \\
\hline
\end{tabular}

OR, odds ratio; 95\% CI, 95\% confidence interval; EGJ, esophagogastric junction. 
results, we found that 15 out of 16 patients (94\%) meeting both criteria (EGJ distensibility at $30 \mathrm{~mL}<2.3 \mathrm{~mm}^{2} / \mathrm{mmHg}$ and acid exposure time $<11 \%$ ) had normalized AET at 6 months follow-up (Fig. 2).

\section{Clinical Treatment Outcome}

In $59 \%$ of patients, an improvement in GERD-HRQL score of at least $50 \%$ was found whereas $41 \%$ of patients had less improvement or even deterioration in symptom scores at 6 months follow-up compared to baseline off PPIs. No differences were found between patients with and without a good clinical response with regard to EndoFLIP and 24-hour pH-impedance parameters both preoperative and at 6 months follow-up. In contrast to objective treatment outcome, preoperative EGJ distensibility and preoperative AET were no predictors of clinical treatment outcome after TIF.

\section{Esophagogastric Junction Distensibility 6 Months After Transoral Incisionless Fundoplication}

In 25 patients, EndoFLIP measurements were repeated direct postoperative and at 6 months follow-up. In the direct postoperative state, EGJ distensibility had decreased from 2.0 (1.2-3.3) to $1.4(1.0-2.2) \mathrm{mm}^{2} / \mathrm{mmHg}(P=0.014)$ at the $30 \mathrm{~mL}$ distension volume. At 6 months follow-up, no change in EGJ distensibility was found compared to baseline (Table 4). Although patients with normalized AET from this subgroup $(\mathrm{n}=14)$ had a lower preoperative EGJ distensibility compared to patients with

Table 4. EndoFLIP Parameters Preoperative, Direct Postoperative and 6 Months After Transoral Incisionless Fundoplication $(\mathrm{N}=25)$

\begin{tabular}{llll}
\hline & Preoperative & Postoperative & $\begin{array}{c}\text { Six months } \\
\text { follow-up }\end{array}$ \\
\hline $\begin{array}{l}\text { EGJ distensibility } \\
\left(\mathrm{mm}^{2} / \mathrm{mmHg}\right)\end{array}$ & & & \\
$\quad 20 \mathrm{~mL}$ & $1.6(1.2-2.4)$ & $1.3(0.8-1.6)^{\mathrm{a}}$ & $1.4(1.0-1.6)$ \\
$30 \mathrm{~mL}$ & $2.0(1.2-3.3)$ & $1.4(1.0-2.2)^{\mathrm{a}}$ & $2.2(1.5-3.0)$ \\
$\mathrm{CSA}\left(\mathrm{mm}^{2}\right)$ & & & \\
$20 \mathrm{~mL}$ & $23(20-29)$ & $20(16-22)$ & $21(20-26)$ \\
$30 \mathrm{~mL}$ & $35(27-49)$ & $36(21-42)$ & $41(27-60)$ \\
Pressure $(\mathrm{mmHg})$ & & & \\
$20 \mathrm{~mL}$ & $14(11-17)$ & $16(14-19)^{\mathrm{a}}$ & $16(15-19)$ \\
$30 \mathrm{~mL}$ & $17(14-24)$ & $21(19-25)^{\mathrm{a}}$ & $20(16-25)$ \\
\hline
\end{tabular}

EGJ, esophagogastric junction; CSA, cross sectional area.

Values are presented as median (IQR).

${ }^{\mathrm{a}} P<0.05$ (preoperative vs postoperative) persistent abnormal AET (1.5 [1.1-2.3] vs 3.0 [1.6-4.3] $\mathrm{mm}^{2} /$ $\mathrm{mmHg}, P<0.05$ ), both groups reached a comparable EGJ distensibility direct postoperative $(1.6[1.0-2.4]$ vs $1.3[0.9-2.1]$ $\left.\mathrm{mm}^{2} / \mathrm{mmHg}, P=0.311\right)$ and at 6 months follow-up (2.0 [1.5-2.8] vs $\left.2.2[1.4-4.0] \mathrm{mm}^{2} / \mathrm{mmHg}, P=0.935\right)$.

\section{Esophagogastric Junction Distensibility and Gastroesophageal Reflux}

Preoperative EGJ distensibility $(30 \mathrm{~mL})$ was weakly correlated with acid exposure time $(r=0.345, P<0.05)$ (Fig. 3) and number of liquid $(r=0.389, P<0.05)$, acid $(r=0.325, P=$ $0.053)$ and proximal reflux episodes $(r=0.474, P<0.01)$ at 6 months follow-up. In contrast, no correlation was found between preoperative EGJ distensibility and AET or number of reflux episodes prior to TIF. Furthermore, no association was found between objective treatment outcome (ie, normalization of acid exposure time) and clinical treatment outcome (ie, $\geq 50 \%$ improvement in GERD-HRQL score) after TIF ( $P=0.868)$.

\section{Discussion}

Restoration of the incompetence of the EGJ by endoscopic or surgical techniques will allow symptom improvement, particularly in GERD patients unresponsive to drug therapy. One of the newer endoscopic approaches aimed to improve EGJ competence is transoral incisionless fundoplication (TIF) with the Esophyx device. One hypothesis states that the TIF procedure restores the gastroesophageal junction by rotating the fundus around the esophagus and creating a partial fundoplication, ${ }^{20}$

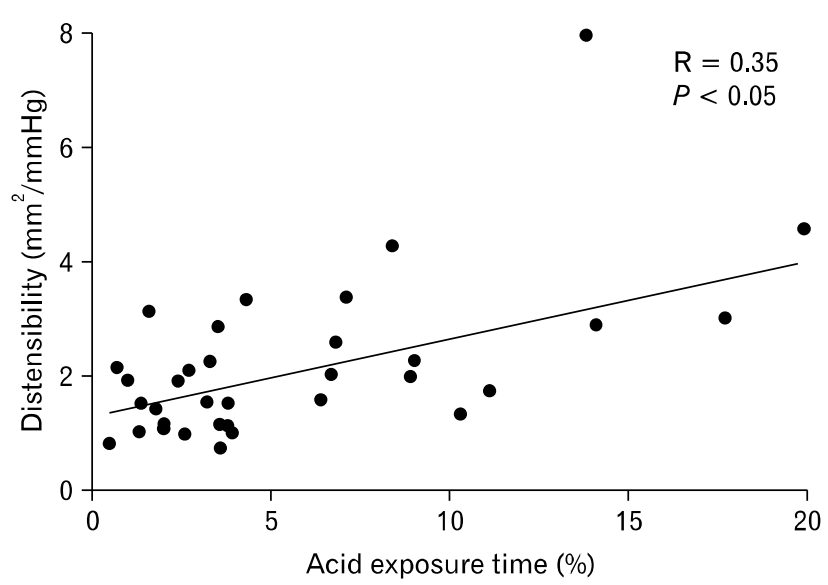

Figure 3. Correlation between preoperative esophagogastric junction (EGJ) distensibility $(30 \mathrm{~mL})$ and acid exposure time at 6 months after transoral incisionless fundoplication. 
thereby restoring EGJ distensibility with reduction in the volume of refluxate since flow across the EGJ is proportional to the opening diameter raised to the fourth power. ${ }^{12}$ The EndoFLIP technique is a new method to assess EGJ distensibility. A previous study described the safety of the use of the EndoFLIP before and direct after endoluminal fundoplication, and in the present study no complications occurred. ${ }^{21}$

In order to predict the responsiveness to TIF already in the preoperative state, we assessed EGJ distensibility using the EndoFLIP technique. We hypothesized that patients with low preoperative EGJ distensibility will have better EGJ competence compared to patients with high EGJ distensibility. Transoral incisionless fundoplication might be able to improve a slightly disturbed EGJ resulting in more competent functioning of the EGJ with consequently less esophageal acid exposure, whereas TIF might be unable to restore EGJ integrity in case of a severely impaired gastroesophageal junction. Our findings confirm the hypothesis by the outcome of a higher percentage of patients with normal gastroesophageal flap valve grade in patients with normalized AET compared to patients with persistent abnormal AET at 6 months follow-up. However, EGJ distensibility was not significantly different 6 months following TIF. The question arises why the decrease in AET and improvement in gastroesophageal flap valve grade is not associated with a change in EGJ distensibility at 6 months after TIF. This could be related to methodological issues of measuring EGJ distensibility. We have to consider that EndoFLIP results are mean values of a 30 -second dynamic measurement. Distensibility of the EGJ is affected by several factors including tone and contractions of the esophagus, crural diaphragm, gastroesophageal flap valve grade and presence or absence of hiatal hernia. A possible limitation of the study is the fact that we measured EGJ distensibility before and after the TIF procedure while patients were under general anesthesia, whereas EndoFLIP measurements at 6 months follow-up were performed under conscious sedation. Although effects of general anesthesia on EGJ distensibility are considered negligible, we cannot exclude that this variable has affected muscle activity and consequently our data. ${ }^{22}$

A recent study demonstrated that EGJ distensibility assessed with the EndoFLIP technique was not able to distinguish GERD patients from healthy controls probably as result of the wide range of EGJ distensibility values in healthy controls. ${ }^{15,23,24}$ In addition, we hypothesized that preoperative EGJ distensibility or CSA would correlate with preoperative esophageal AET, as the EGJ opening diameter determines reflux volume. ${ }^{12}$ However, no correlation was found between preoperative EGJ distensibility or CSA and the severity of GERD according to esophageal AET. Therefore, the EndoFLIP technique cannot be used to diagnose GERD or to grade the severity of GERD according to esophageal AET. ${ }^{23}$ Tucker et al suggested that endoluminal measurement of EGJ distensibility with the EndoFLIP technique may not be able to reflect the action or interaction of the different structures of the complex EGJ which might be a potential explanation for the lack of correlation between both EGJ distensibility and AET prior to TIF. ${ }^{23}$ Moreover, Tucker et al found an inverse correlation between EGJ distensibility, CSA and BMI. In the present study, BMI was not correlated to either preoperative EGJ distensibility or CSA, probably due to a lower median $\mathrm{BMI}$ with a narrower range in our participants. ${ }^{23}$

Although no difference was observed in EGJ distensibility following TIF, we did observe significant improvement in acid exposure time in $57 \%$ of the patients which is comparable to previous publications. ${ }^{8,25}$ Therefore, we suggest that changes in other factors like transient LES relaxations and the acid pocket may contribute to the antireflux effect of the TIF procedure, and probably may explain the observed difference between patients with normalized AET and patients with persistent abnormal AET at 6 months follow-up. ${ }^{26,27}$

Patient selection is crucial in order to achieve optimal treatment outcome after TIF as normalization of AET was only present in $57 \%$ of patients. According to literature data, GERD patients with small hiatal hernia $(\leq 2 \mathrm{~cm})$ and normal gastroesophageal flap valve grade (Hill I-II) prior to TIF are considered best candidates. ${ }^{6,10}$ In the present study, gastroesophageal flap valve grade and presence or absence of hiatal hernia prior to TIF were not associated with normalization of AET after TIF. However, our results show that lower preoperative EGJ distensibility and moderately increased preoperative AET are associated with better objective treatment outcome after TIF (ie, normalization of AET), whereas patients with high preoperative distensibility and prolonged AET appear to be better candidates for laparoscopic fundoplication. Based on our results, we suggest that patients with preoperative EGJ distensibility $<2.3 \mathrm{~mm} / \mathrm{mmHg}$ and preoperative AET $<11 \%$ have the best objective treatment outcome. Recently, a comparable cutoff value of $10 \%$ for preoperative AET was suggested. ${ }^{25}$ However, it should be taken into account that this is the first study reporting on preoperative EGJ distensibility as predictive factor for objective treatment outcome of TIF. Our sample size is small and additional data are needed to confirm our results and optimize cut-off values. 
Furthermore, we have to consider that an effective treatment for GERD is not only expected to reduce AET, heal esophagitis and prevent the development of chronic complications, but also to relieve GERD symptoms. In contrast to objective treatment outcome, preoperative EGJ distensibility could not predict clinical treatment outcome after TIF. In addition, no association was found between objective (ie, normalization of acid exposure time) and clinical (ie, $\geq 50 \%$ improvement in GERD-HRQL score) treatment outcome. Although the first sham controlled trials are awaited, we hypothesize that one possible explanation for this apparent discrepancy might be the placebo effect as both patients with decreased and unaltered AET showed symptom improvement. ${ }^{28,29}$ In addition, Trad et al described a lack of symptom improvement in patients with persistent pathological AET after acid suppressive therapy, whereas patients with persistent pathological AET after TIF had improved symptom scores. ${ }^{25}$ Moreover, we have to take into account that symptom perception in GERD patients is a complex pathophysiological mechanism influenced by multiple factors including reflux characteristics (ie, acidity, proximal extent, and composition of the refluxate), hypersensitivity and mucosal barrier function. ${ }^{30,31}$ In the present study, we evaluated reflux characteristics according to 24-hour $\mathrm{pH}$-impedance monitoring, whereas we did not objectively assess changes in (hyper)sensitivity and mucosal barrier function after TIF. This might at least partially explain the lack of association between objective and clinical treatment outcome after TIF, especially as previous studies described discrepancies between the severity of esophageal acid exposure and reported symptoms. ${ }^{30,32}$

In addition, our study had other limitations. We assessed the predictive effect of EGJ distensibility for medium term treatment outcome based on acid exposure time and symptom scores 6 months after TIF. Gastroesophageal reflux disease is however a chronic disorder and assessment of the predictive value of preoperative EGJ distensibility for long-term follow-up is necessary. Furthermore, we have to take into account practical and financial aspects of the EndoFLIP technique especially as assessment of AET is part of the preoperative work-up of GERD patients. Measurement of EGJ distensibility with the EndoFLIP device is a relatively new method and the precise role of this technique in the diagnosis and management of upper gastrointestinal diseases is still unknown. Therefore, the EndoFLIP is not a routine technique and only available in a limited number of GI centers, despite the fact that the device is commercially available. Although EndoFLIP offers the possibility to acquire assessment of EGJ distensibility in a more practical method compared to barostat, we have to consider that measurement of EGJ distensibility with the EndoFLIP technique remains an invasive procedure which may be associated with discomfort in patients. ${ }^{33}$ Finally, we have to take into account additional costs of this technique as both the mobile recording device and single-use catheters are expensive.

Taken our results and arguments into consideration, no evidence has been provided for the use of the EndoFLIP technique in GERD patients either in the preoperative diagnostic work-up or in the post-procedure evaluation. However, we cannot exclude that the EndoFLIP device may have additional value in the follow-up of patients with postoperative symptoms (eg, dysphagia) after antireflux procedures or for assessment of treatment efficacy in achalasia patients. ${ }^{24}$

In conclusion, preoperative esophagogastric junction (EGJ) distensibility and preoperative esophageal acid exposure time were independent predictors for objective treatment outcome after transoral incisionless fundoplication in GERD patients according to esophageal acid exposure time, whereas preoperative EGJ distensibility was not able to predict subjective, clinical treatment outcome. Moreover, EGJ distensibility was not different at 6 months follow-up compared to baseline. According to our data, we conclude that the EndoFLIP technique has no added value either in the preoperative diagnostic work-up or in the post-procedure evaluation of endoluminal antireflux therapy.

\section{References}

1. Vakil N. Review article: the role of surgery in gastro-oesophageal reflux disease. Aliment Pharmacol Ther 2007;25:1365-1372.

2. Lundell L, Miettinen P, Myrvold HE, et al. Seven-year follow-up of a randomized clinical trial comparing proton-pump inhibition with surgical therapy for reflux oesophagitis. Br J Surg 2007;94: 198-203.

3. Freston JW, Triadafilopoulos G. Review article: approaches to the long-term management of adults with GERD-proton pump inhibitor therapy, laparoscopic fundoplication or endoscopic therapy? Aliment Pharmacol Ther 2004;19(suppl 1):35-42.

4. Louis H, Deviere J. Ensocopic-endoluminal therapies. A critical appraisal. Best Pract Res Clin Gastroenterol 2010;24:969-979.

5. Cadiere GB, Van Sante N, Graves JE, Gawlicka AK, Rajan A. Two-year results of a feasibility study on antireflux transoral incisionless fundoplication using EsophyX. Surg Endosc 2009;23:957-964.

6. Cadiere GB, Buset M, Muls V, et al. Antireflux transoral incisionless fundoplication using EsophyX: 12-month results of a prospective multicenter study. World J Surg 2008;32:1676-1688.

7. Frazzoni M, Conigliaro R, Manta R, Melotti G. Reflux parameters as modified by EsophyX or laparoscopic fundoplication in refractory GERD. Aliment Pharmacol Ther 2011;34:67-75.

8. Bell RC, Mavrelis PG, Barnes WE, et al. A prospective multicenter 
registry of patients with chronic gastroesophageal reflux disease receiving transoral incisionless fundoplication. J Am Coll Surg 2012; 215:794-809.

9. Testoni PA, Vailati C, Testoni S, Corsetti M. Transoral incisionless fundoplication (TIF 2.0) with EsophyX for gastroesophageal reflux disease: long-term results and findings affecting outcome. Surg Endosc 2012;26:1425-1435.

10. Witteman BP, Strijkers R, de Vries E, et al. Transoral incisionless fundoplication for treatment of gastroesophageal reflux disease in clinical practice. Surg Endosc 2012;26:3307-3315.

11. Ghosh SK. Biomechanics of the esophagogastric junction in gastroesophageal reflux disease. Curr Gastroenterol Rep 2008;10:246-251.

12. Pandolfino JE, Shi G, Curry J, Joehl RJ, Brasseur JG, Kahrilas PJ. Esophagogastric junction distensibility: a factor contributing to sphincter incompetence. Am J Physiol Gastrointest Liver Physiol 2002;282:G1052-G1058.

13. Pandolfino JE, Curry J, Shi G, Joehl RJ, Brasseur JG, Kahrilas PJ. Restoration of normal distensive characteristics of the esophagogastric junction after fundoplication. Ann Surg 2005;242:43-48.

14. Blom D, Bajaj S, Liu J, et al. Laparoscopic Nissen fundoplication decreases gastroesophageal junction distensibility in patients with gastroesophageal reflux disease. J Gastrointest Surg 2005;9:13181325.

15. Kwiatek MA, Pandolfino JE, Hirano I, Kahrilas PJ. Esophagogastric junction distensibility assessed with an endoscopic functional luminal imaging probe (EndoFLIP). Gastrointest Endosc 2010;72: 272-278.

16. Velanovich V. The development of the GERD-HRQL symptom severity instrument. Dis Esophagus 2007;20:130-134.

17. Rothstein R, Filipi C, Caca K, et al. Endoscopic full-thickness plication for the treatment of gastroesophageal reflux disease: a randomized, sham-controlled trial. Gastroenterology 2006;131:704-712.

18. Hill LD, Kozarek RA. The gastroesophageal flap valve. J Clin Gastroenterol 1999;28:194-197.

19. McMahon BP, Frokjaer JB, Kunwald P, et al. The functional lumen imaging probe (FLIP) for evaluation of the esophagogastric junction. Am J Physiol Gastrointest Liver Physiol 2007;292:G377G384.

20. Bell RC, Cadière GB. Transoral rotational esophagogastric fundoplication: technical, anatomical, and safety considerations. Surg Endosc 2011;25:2387-2399.

21. Hoppo T, McMahon BP, Witteman BP, et al. Functional lumen imaging probe to assess geometric changes in the esophagogastric junction following endolumenal fundoplication. J Gastrointest Surg 2011;15:1112-1120.

22. Nathanson LK, Brunott N, Cavallucci D. Adult esophagogastric junction distensibility during general anesthesia assessed with an endoscopic functional luminal imaging probe (EndoFLIP(R)). Surg Endosc 2012;26:1051-1055.

23. Tucker E, Sweis R, Anggiansah A, et al. Measurement of esophago-gastric junction cross-sectional area and distensibility by an endolumenal functional lumen imaging probe for the diagnosis of gastroesophageal reflux disease. Neurogastroenterol Motil 2013;25: 904-910.

24. Rohof WO, Hirsch DP, Kessing BF, Boeckxstaens GE. Efficacy of treatment for patients with achalasia depends on the distensibility of the esophagogastric junction. Gastroenterology 2012;143:328-335.

25. Trad KS, Barnes WE, Simoni G, et al. Transoral incisionless fundoplication effective in eliminating GERD symptoms in partial responders to proton pump inhibitor therapy at 6 months: the TEMPO randomized clinical trial. Surg Innov 2014;22:26-40.

26. Rinsma NF, Smeets FG, Bruls DW, et al. Effect of transoral incisionless fundoplication on reflux mechanisms. Surg Endosc 2013; 28:941-949.

27. Herbella FA, Vicentine FP, Del Grande JC, Patti MG. Postprandial proximal gastric acid pocket in patients after laparoscopic Nissen fundoplication. Surg Endosc 2011;25:3198-3201.

28. Hogan WJ. Clinical trials evaluating endoscopic GERD treatments: is it time for a moratorium on the clinical use of these procedures? Am J Gastroenterol 2006;101:437-439.

29. Pearl JP, Marks JM. Endolumenal therapies for gastroesophageal reflux disease: are they dead? Surg Endosc 2007;21:1-4.

30. Weijenborg PW, Bredenoord AJ. How reflux causes symptoms: Reflux perception in gastroesophageal reflux disease. Best Pract Res Clin Gastroenterol 2013;27:353-364.

31. Harnett KM, Rieder F, Behar J, Biancani P. Viewpoints on acid-induced inflammatory mediators in esophageal mucosa. J Neurogastroenterol Motil 2010;16:374-388.

32. Stein HJ, Hoeft S, DeMeester TR. Functional foregut abnormalities in Barrett's esophagus. J Thorac Cardiovasc Surg 1993;105: 107-111.

33. Verlaan T, Rohof WO, Bredenoord AJ, Eberl S, Rösch T, Fockens P. Effect of peroral endoscopic myotomy on esophagogastric junction physiology in patients with achalasia. Gastrointest Endosc 2013; 78:39-44. 